\title{
Y-glutamyl transpeptidase deficiency caused by a large homozygous intragenic deletion in GGT1
}

\author{
Niklas Darin ${ }^{1} \cdot$ Karin Leckström² $\cdot$ Per Sikora $^{3,4} \cdot$ Julia Lindgren² $^{2}$ Gabriella Almén $^{2} \cdot$ Jorge Asin-Cayuela $^{2}$
}

Received: 15 September 2017 / Revised: 21 December 2017 / Accepted: 1 February 2018 / Published online: 26 February 2018

(c) European Society of Human Genetics 2018

\begin{abstract}
$\gamma$-Glutamyl transpeptidase deficiency (glutathionuria, OMIM 231950) is a rare disease, with only six patients reported in the literature, although this condition has probably been underdiagnosed due the difficulty to routinely analyze glutathione in clinical samples and to the fact that no genetic defect has been coupled to the disease so far. We report two siblings with mild psychomotor developmental delay and mild neurological symptoms, who presented a markedly increased excretion of glutathione in urine and a very low $\gamma$-glutamyl transpeptidase activity in serum. Whole-genome sequencing revealed the presence of a $16.9 \mathrm{~kb}$ homozygous deletion in GGT1, one of the genes encoding enzymes with $\gamma$-glutamyl transpeptidase activity in the human genome. Close analysis revealed the presence of a $13 \mathrm{bp}$ insertion at the deletion junction. This is the first report of a genetic variant as the cause of glutathionuria. In addition, genetic characterization of the patients' parents and a healthy sibling has provided direct genetic evidence regarding the autosomal recessive nature of this disease.
\end{abstract}

\section{Introduction}

Glutathionuria (OMIM 231950) is caused by a defect in $\gamma$ glutamyl transpeptidase ( $\gamma$-GT, EC 2.3.2.2.), the enzyme that cleaves the $\gamma$-glutamyl bond of glutathione (GSH, $\gamma$-Lglutamyl-L-cysteinylglycine) to produce cysteinylglycine and glutamate [1]. GSH is the most abundant non-protein thiol compound in cells and as such it has an important role in antioxidant defense, xenobiotic detoxification, maintenance of redox homeostasis, and cell signaling via $S$ -

Electronic supplementary material The online version of this article (https://doi.org/10.1038/s41431-018-0122-6) contains supplementary material, which is available to authorized users.

$\triangle$ Jorge Asin-Cayuela

jorge.asin.cayela@vgregion.se

1 Department of Pediatrics, Institute of Clinical Sciences, Sahlgrenska Academy at University of Gothenburg, Gothenburg, Sweden

2 Department of Clinical Chemistry, Sahlgrenska University Hospital, Gothenburg, Sweden

3 Department of Laboratory Medicine, Sahlgrenska University Hospital, Gothenburg, Sweden

4 Department of Clinical Genomics, Gothenburg Science for Life Laboratories, Gothenburg, Sweden glutathionylation of proteins such as receptors, protein kinases, and transcription factors. GSH is also a substrate for the synthesis of eicosanoids. For excellent extensive reviews on the biological functions of GSH, see references $[2,3$,$] .$

The first patient with glutathionuria was described in 1971, although a previous possible case is cited [4]. To the present day, only six patients from four families have been reported [5-11]. Most patients presented involvement of the central nervous system in the form of moderate mental retardation, behavioral disturbance, and, in one case, seizures [8]. Marfanoid features were observed in two siblings [11]. Age of diagnosis ranged from the second to the sixth decade of life. In none of these cases was the genetic cause of glutathionuria identified. See Table 1 for a summary of clinical and biochemical features of all patients reported in the literature.

Several genes are potentially involved in the expression of enzymes with $\gamma$-GT activity in humans [12]. GGT1, the best characterized, encodes an extracellular enzyme anchored to the plasma membrane of cells that is highly efficient in the cleavage of the $\gamma$-glutamyl bond of oxidized and reduced GSH, as well as leukotriene $\mathrm{C}_{4}$, whereas the product of GGT5 acts mostly on reduced GSH and leukotriene $\mathrm{C}_{4}$, albeit less efficiently than the product of $G G T 1$ [13]. GGT2 expresses an inactive protein, due to its inability to undergo the autoproteolytic cleavage to produce a large 
and a small subunit, which is essential for the catalytic activity of $\gamma$-GTs [14]. Very little is known about GGT6 and GGT7. The latter, highly expressed in the brain, has been recently involved in the progression of glioblastoma [15]. Two other genes, GGTLC1 and GGTLC2, have the potential to encode the light chain of $\gamma$-GT. In addition, according to NCBI (National Center for Biotechnology Information), four pseudogenes sharing homology with $G G T 1$ are present in the human genome, namely GGT3P, GGTLC5P, $G G T 8 P$, and GGT4P.

Here we report two siblings with glutathionuria caused by a large homozygous deletion in GGT1. This is, to our knowledge, the first report that links a genetic defect to this condition.

\section{Material/subjects and methods}

\section{Patients' clinical description}

\section{Patient 1}

This boy is the third child to healthy parents of Turkish origin. He has a healthy older brother and an affected older sister (Patient 2). He was born at term after a normal gestation. Birth weight was $2,840 \mathrm{~g}(-2 \mathrm{SD})$, length was $48 \mathrm{~cm}(-1.5 \mathrm{SD})$, and head circumference was $34 \mathrm{~cm}$ (1.5 SD). The APGAR score was 9-10-10 and the perinatal period was uncomplicated. The psychomotor development was delayed and he started to say single words and to walk unsupported at 2 years of age. There has been no regression. At the age of 10 years, the patient was first referred to us for investigations because of suspected mental retardation. Psychological testing at 11 years of age revealed moderate learning disability and the boy has attended special school. He has been healthy except for day and nighttime urinary incontinence, mild constipation, and treatment because of atopic rhinoconjunctivitis, eczema, and asthma bronchiale. Clinical examination at 11 years of age revealed mild hypotelorism and low-seated ears. He had mild ataxia upon walking, intention tremor, mild dysdiadochokinesis, and mild hyperreflexia $(3+)$ but there was no clonus or spasticity, and Babinski's sign was negative. The weight was $43.9 \mathrm{~kg}(+1 \mathrm{SD})$, height was $151 \mathrm{~cm}(+1 \mathrm{SD})$, and head circumference $52.5 \mathrm{~cm}(-0.8 \mathrm{SD})$. Magnetic resonance imaging (MRI) of the brain at the same age showed agenesis of corpus callosum and multiple cortical heterotopias (See Fig. 1).

\section{Patient 2}

This girl is the older sister to patient 1 . She was born at term after a normal gestation. Birth weight was $3330 \mathrm{~g}(-0.5$
SD), length was $50 \mathrm{~cm}( \pm 0 \mathrm{SD})$, and head circumference was $34 \mathrm{~cm}(-1 \mathrm{SD})$. The APGAR score was $10-10-10$ and the perinatal period was uncomplicated. The psychomotor development was delayed and she started to say single words and to walk unsupported at 2 years of age. Psychological testing at 7 years of age revealed a mild learning disability and the girl has attended special school. During childhood, she was healthy except for day and nighttime urinary incontinence, which disappeared during adolescence. At 7 years of age, weight was $22.9 \mathrm{~kg}( \pm 0 \mathrm{SD})$, height was $122.3 \mathrm{~cm}( \pm 0 \mathrm{SD})$, and the head circumference was $50 \mathrm{~cm}(-1 \mathrm{SD})$. She was first investigated by us at 25 years of age as a consequence of the findings observed in her brother (patient 1). She was at that time generally healthy. Clinical examination was normal except for a mild action tremor and mild hyperreflexia $(3+)$ but there was no clonus or spasticity, and Babinski's sign was negative. MRI of the brain and ophthalmological investigations were normal.

\section{Detection of GSH in urine}

Amino acids in urine were analyzed by cation-exchange chromatography followed by ninhydrin derivatization and spectrophotometric detection on an AminoTac JLC-500/V analyzer (JEOL, Croissy Sur Seine, France) essentially as described [16]. Hydrolysis on vapor-phase was carried out as described [17] by incubating a urine sample with $6 \mathrm{~N}$ $\mathrm{HCl}$ for $24 \mathrm{~h}$ at $110^{\circ} \mathrm{C}$ on a Pico-Tag workstation (Waters, Milford, MA, USA).

\section{Determination of $\mathrm{Y}$-GT activity in serum}

The activity of $\gamma$-GT in serum was analyzed spectrophotometrically on a COBAS c 501 autoanalyzer (Roche Diagnostics Scandinavia AB, Solna, Sweden) following the manufacturer's instructions.

\section{Whole-genome sequencing and Sanger verification}

DNA obtained from blood was extracted using QIAamp DNA Blood Mini Kit (Qiagen Benelux BV, Venlo, The Netherlands). One microgram of purified DNA was sent for sequencing at the Clinical Genomics facility at the Science for Life Laboratory, Solna, Sweden, for whole-genome sequencing (WGS) on the Illumina Xten at $30 \times$ coverage. Sequencing returned $\sim 139 \mathrm{~Gb}$, corresponding to a $44.78 \times$ coverage before quality trimming. The sample was then trimmed, mapped, and variant called using CLC Biomedical Server (Qiagen Aarhus, Aarhus, Denmark) with a workflow developed at the Clinical Genomics Gothenburg facility. The data set was trimmed (limit 0.02, minimum length 30 , max number of ambiguous bases 2) and then mapped 
Fig. 1 MRI of the brain of patient 1 at 11 years of age. Sagittal T1-weighted (TR/TE $=$ 420/8.6 ms) a and axial T2 weighted $(\mathrm{TR} / \mathrm{TE}=4050 / 98$ ms) b images showing the absence of corpus callosum (indicated by arrows in a) and secondary paralleled lateral ventricles with widened posterior horns and reduction of periventricular white matter (indicated by arrows in b). Coronal T1 (TR/TE $=1160 / 4.2$ ms) images c, d show heterotopias in relation to the left and right posterior horns of the lateral ventricles (indicated by arrows $\mathbf{c}$ and $\mathbf{d}$, respectively)

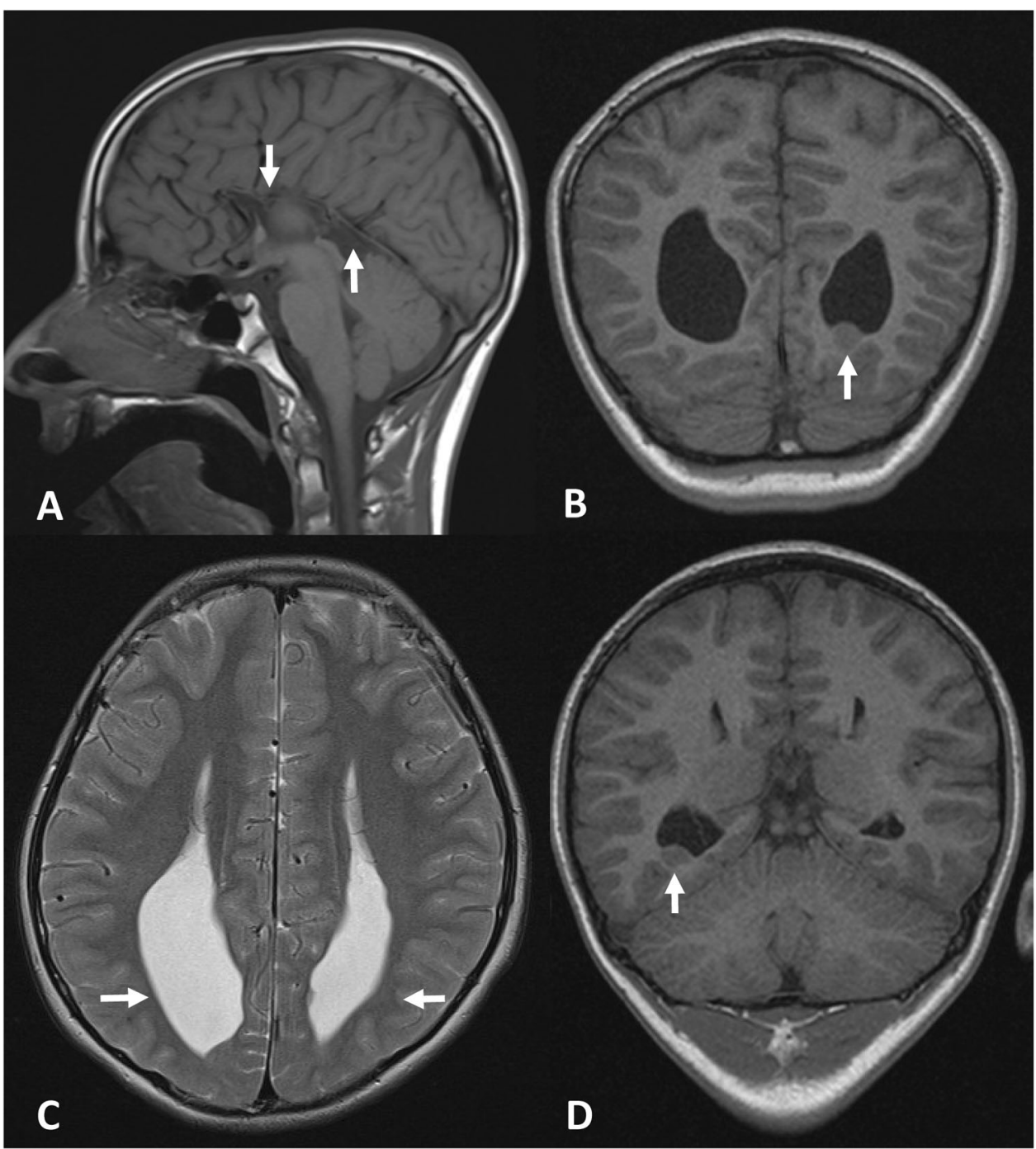

against the hg19 reference genome (98.86\% total reads mapped, $91.87 \%$ total reads in pairs). After mapping, indels were called ( $p$-value 0.001 , max mismatches 3 , minimum reads 2) and the mapping was locally realigned in three passes using the indel data. Variants were generated using the CLC Fixed Ploidy variant caller (minimum coverage 7, minimum count 2 , minimum frequency $15 \%$ ) and the results filtered against RefSeq HG19 coding sequences. Variants were analyzed with Ingenuity Variant Analysis software (Qiagen Aarhus, Aarhus, Denmark) and Integrative Genomics Viewer (IGV) visualization tool [18]. The deletion identified by WGS was verified by Sanger sequencing using primers amplifying the genomic region harboring the deletion in chromosome 22 (including parts of GGT1). Sequencing analysis was performed using an ABI PRISM 3130XL Genetic Analyzer and the BigDye Terminator v.1.1 Cycle Sequencing Kit (Applied Biosystems, ThermoFisher Scientific, Waltham, MA, USA). Primer sequences and PCR conditions are available upon request.
The deletion reported in this manuscript has been submitted to the Leiden Open Variation Database (https://data bases.lovd.nl/shared/variants/0000236419) with ID: GGT1_000001.

\section{Results}

The analysis of amino acids in urine revealed the presence of three peaks of unclear origin with retention times close to aspartate, glycine, and citrulline respectively (see peaks marked with asterisks in Fig. 2a). This pattern was essentially identical in both patients. On close observation, the first two peaks had retention times consistent with reduced and oxidized GSH, respectively (Fig. 2b), whereas the peak close to citrulline could correspond to a thio-compound previously described in a patient with glutathionuria [11]. Hydrolysis with $6 \mathrm{~N} \mathrm{HCl} \mathrm{(Fig.} \mathrm{2c)} \mathrm{resulted} \mathrm{in} \mathrm{the} \mathrm{dis-}$ appearance of all three peaks and a concomitant increase of 

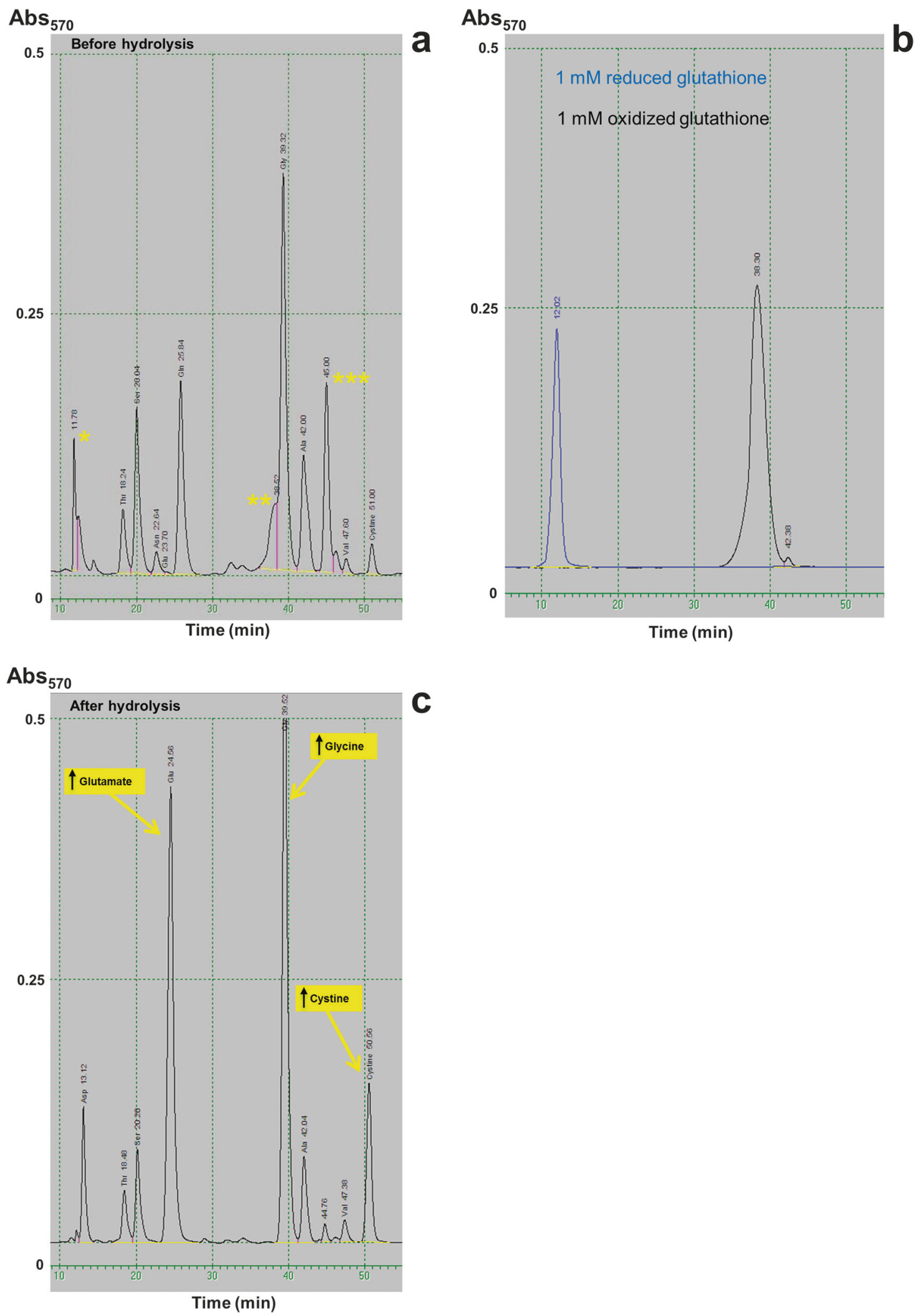

Fig. 2 Analysis of amino acids in urine. a Direct analysis revealed the presence of three peaks of unclear identity (marked with asterisks). Two of them $(*$ and $* *$ ) have the same retention time as reduced and oxidized glutathione, respectively, as show in $\mathbf{b}$. The third one $(* * *)$ has a retention time consistent with a thiol derivative previously

described in a patient with glutathionuria [11]. $\mathbf{c}$ Hydrolysis in $6 \mathrm{~N} \mathrm{HCl}$ eliminates all three peaks and produces an increase of glutamate, glycine, and cystine, the expected hydrolysis products of glutathione in non-reducing conditions 
Fig. 3 Visualization of WGS data covering GGT1. a Plot of paired reads from patient 1 indicates the presence of a homozygous deletion in GGT1. The deletion includes the first coding exon of all isoforms of GGT1 as well as several noncoding exons. All control samples tested presented a low coverage region that corresponds to a low complexity DNA sequence. $\mathbf{b}$ Mapping of soft-clipped ends defines the deletion between positions Chr22:24,992,587 and

Chr22:25,009,579. Paired reads flanking the deletion are depicted in matching colours. c. Alignment of soft-clipped ends from the centromeric breakpoint (upper panel) and telomeric breakpoint (middle panel) reveals the additional presence of a $13 \mathrm{bp}$ duplication. Sanger sequencing of the deletion junction (lower panel) confirms the WGS findings
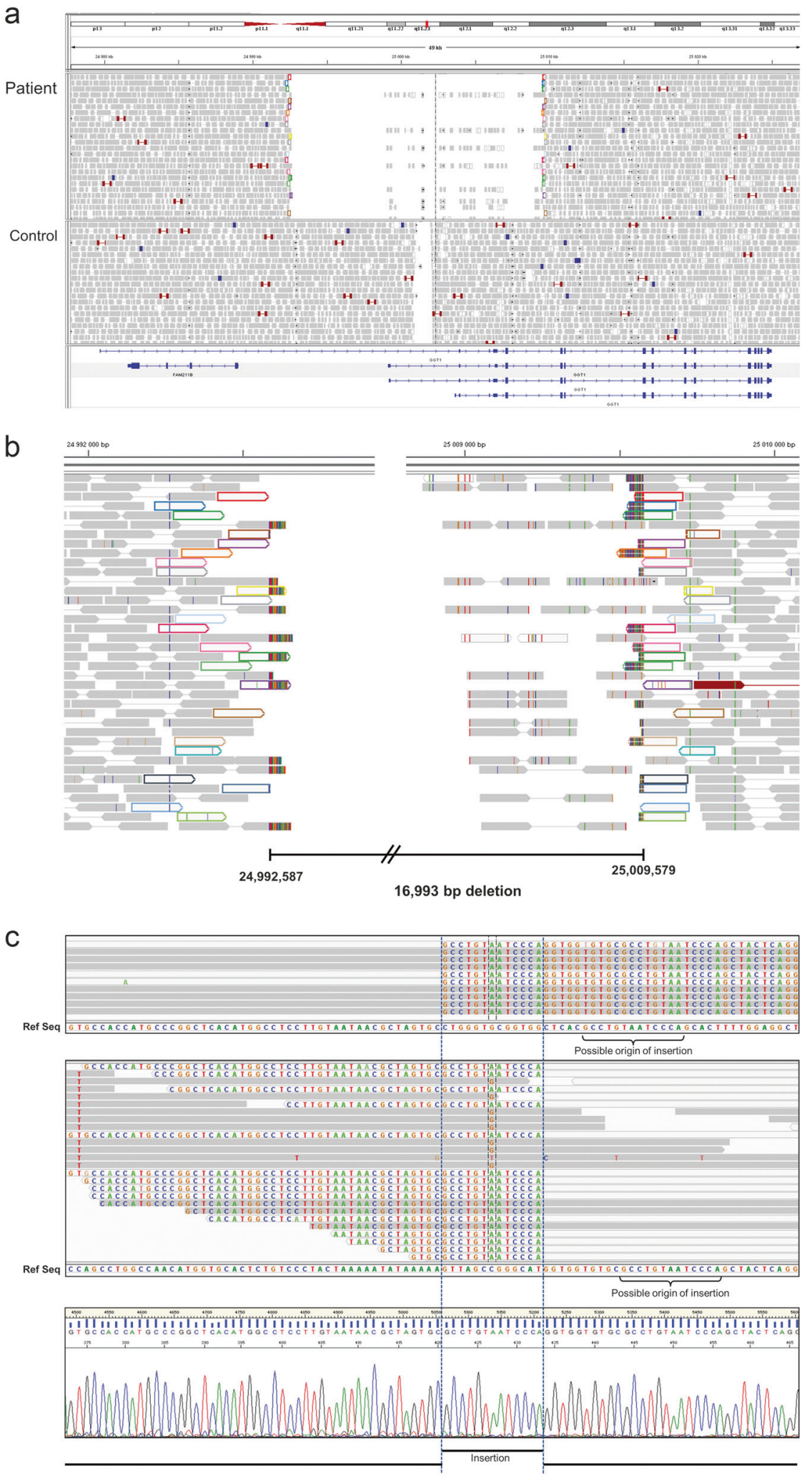

$24,992,587 \quad 25,009,579$

glutamate, glycine, and cystine. It is well described that hydrolysis in $6 \mathrm{~N} \mathrm{HCl}$ completely transforms glutamine to glutamate (as well as asparagine to aspartate), and that cysteine is oxidized to cystine in non-reducing conditions. The inferred increases of glutamate and cysteine (in the form of cystine) after hydrolysis are nearly equimolar $(\sim 1$ 
$\mathrm{mmol} / \mathrm{l}$; we could not quantify glycine in the pre-hydrolysis sample due to the interference of oxidized GSH). Taken together, we conclude that both the original pattern of amino acids and the results of the hydrolysis are consistent with glutathionuria. This diagnosis was further supported by analysis of $\gamma$-GT activity in serum, which was under the limit of quantification of the technique in both affected siblings $(<0.05 \mu \mathrm{kat} / \mathrm{l}$, reference ranges: $<0.6$ for patient 1 and $0.15-0.75$ for patient 2). $\gamma$-GT activity of both parents laid within the normal range for their respective ages and
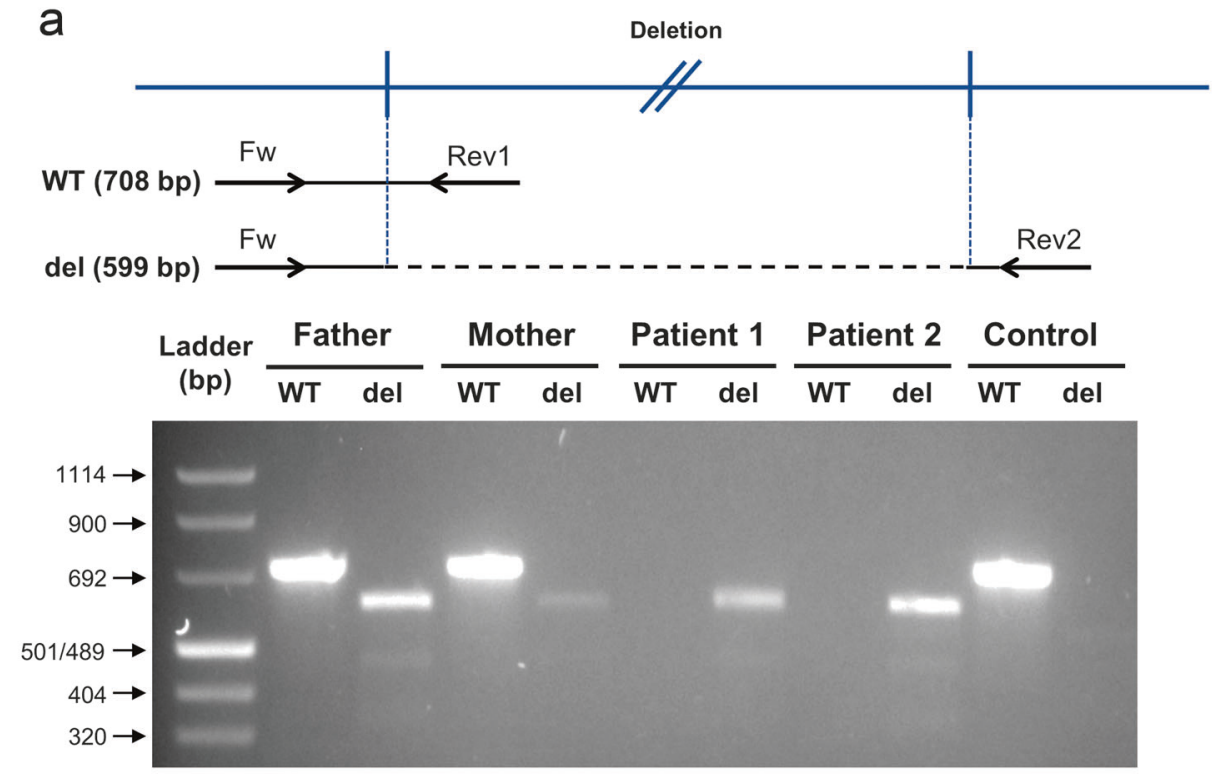

b
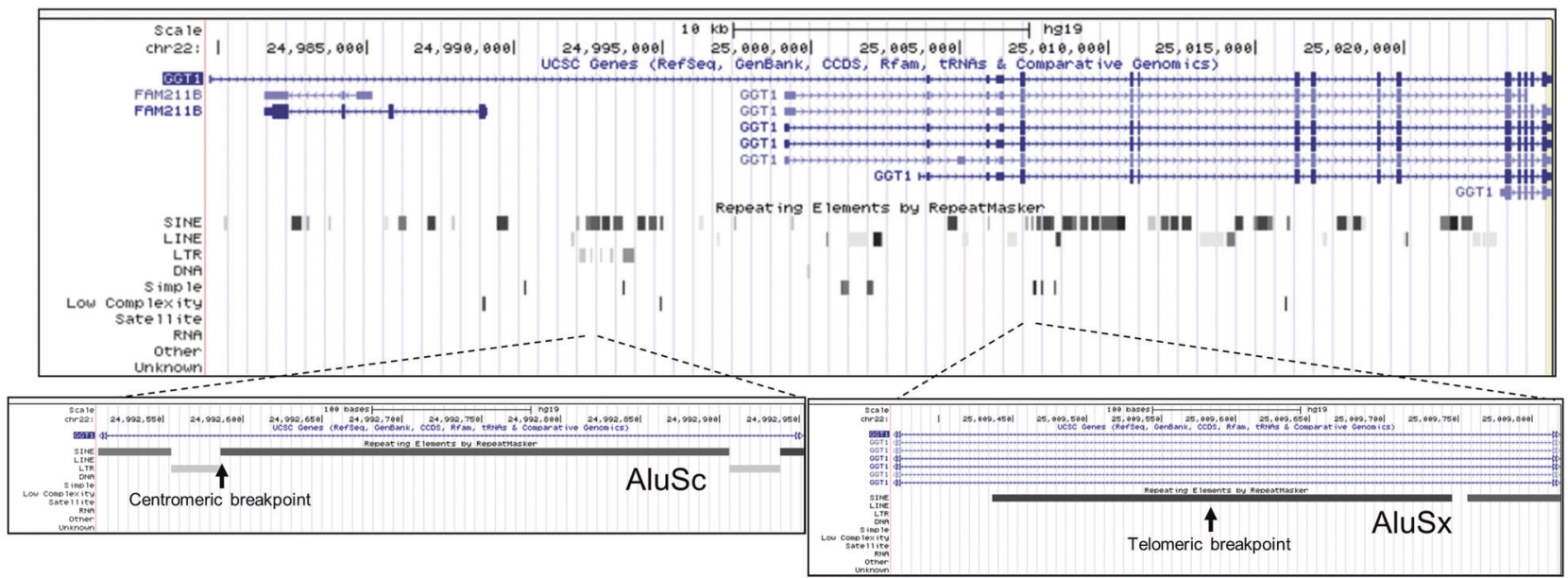

$\downarrow$ 
Fig. 4 a Agarose gel electrophoresis of fragments amplified from the DNA of both patients and their parents using primers designed to give a PCR product in the absence/presence of the deletion. Upper panel: primer pair Fw-Rev1 (WT) is expected to produce a 708 bp product only in the absence of the deletion, whereas primer pair Fw-Rev2 (del) is expected to produce a $599 \mathrm{bp}$ only in the presence of the deletion. Lower panel: DNA from both parents produced products with both primer pairs, indicating that they are heterozygotes for the deletion. DNA from both affected siblings produced a product only with the "del" primer pair, as expected for homozygotes for the deletion, whereas a control sample produced a product from the WT pair only. b Both breakpoints of the deletion are located in Alu elements. Upper panel: UCSC depiction of GGT1. RepeatMaster track shows abundant SINE elements in this gene. Zooming at both breakpoints shows that both are located in Alu elements. Middle panel: ClustalO alignment of the flanking regions of both breakpoints reveals substantial areas of homology, denoted by asterisks. Of special interest is the presence of a region close to both breakpoints that shares high homology to the 26 bp Alu-DEIN sequence, a recombination hotspot. The breakpoints are indicated by arrows. Lower panel: alignment of centromeric and telomeric reference sequences with the sequence obtained at the junction from patient 1 . The $13 \mathrm{bp}$ insertion and its two possible origins are encased in yellow. Horizontal black bars indicate putative microhomology regions. See Supplementary Figure 2 for proposed microhomology-based replication slippage and template-switching events underlying the genomic rearrangement presented here

genders (mother: $0.18 \mu \mathrm{kat} / \mathrm{l}$, reference range: 0.15-1.2; father: $0.28 \mu \mathrm{kat} / \mathrm{l}$, reference range: $0.20-1.9$ ).

In order to confirm the diagnosis we took a genetic approach. As the genetic cause of glutathionuria was not known, we created an in silico panel containing all expressed genes in the human $\gamma$-GT gene family as defined by Heisterkamp et al. [12], namely GGT1, GGT2, GGT5, GGT6, GGT7, GGTLC1, GGTLC2, and GGTLC3, and used WGS as the sequencing platform for patient 1 . Initial analysis did not detect any single nucleotide variants nor small insertions/deletions of potential pathogenic nature in any of the genes included in the panel. However, close inspection using IGV browser indicated the presence of a large homozygous deletion in GGT1, which included the first coding exon of all isoforms (Fig. 3a). Analysis of the softclipped ends (that is, fragments of reads not mapped to the reference sequence) of the reads that cover the junction revealed the exact location of both breakpoints at positions Chr22:24,992,587 (centromeric) and Chr22:25,009,579 (telomeric), respectively (Fig. 3b). In addition, the alignment of both sets of reads revealed the presence of a $13 \mathrm{bp}$ insertion identical to two sequences, each one in the vicinity of each breakpoint (Fig. 3c). As opposed to the centromeric breakpoint, a number of reads at the telomeric breakpoint align to the reference sequence past the breakpoint (see Fig. 3c, central panel). Interestingly, all those reads contain mismatches that can be traced to pseudogenes GGT3P and GGT4P. Consequently, we conclude that those reads are misaligned to GGT1.

$G G T 1$-specific primers flanking the deletion junction gave a PCR product with the expected size (primers Fw and
Rev2 in Fig. 4a). Sequencing of the PCR product confirmed the WGS findings (Fig. 3c, bottom panel). A second set of primers was designed to give a PCR product only in the absence of the deletion (primers Fw and Rev1 in Fig. 4a). DNA from both affected siblings produced a PCR product from the first primer pair but not from the second, whereas DNA from the parents produced PCR products from both primer pairs, as expected for heterozygotes for the deletion. DNA from a control sample produced a PCR product only from the second primer set (Fig. 4a). The healthy brother showed a pattern consistent with heterozygosity for the deletion (data not shown).

In conclusion, WGS data and Sanger sequencing indicate the presence of a homozygous 16,993 bp deletion combined to a $13 \mathrm{bp}$ insertion in GGT1 that removes the first coding exon and several non-coding exons of all isoforms of GGT1.

\section{Discussion}

Despite being a condition already described in the early 70 s, only a few patients with glutathionuria have been reported so far. In addition to this, the fact that some of these patients were detected by screening for amino acid defects in cohorts of mentally retarded individuals or in infants (1 respective 3 in Table 1), that one of them is asymptomatic (3), and that one (4) was diagnosed with a second condition that could account for her clinical picture, raises the question of ascertainment bias being coupled to this condition. The two patients reported here were diagnosed in the process of investigating the cause for their moderate mental retardation, which happens to be the predominant clinical feature in all but one of the patients reported, a condition that perhaps is not always investigated with the diagnostic tools necessary to establish the diagnosis of $\gamma$-GT deficiency. Identification of more cases will help to definitively establish $\gamma$-GT deficiency as the cause of the clinical picture observed in these patients.

One factor that could contribute to explain why the number of patients reported is so low is that GSH is not a metabolite commonly measured in clinical laboratories, and that the identification of GSH with the amino acid analyzers based on ion-exchange chromatography and ninhydrin staining in use today is not straightforward. The presence of extra peaks in the analysis of amino acids, especially in urine, is a common phenomenon and often corresponds to exogenous substances with no direct diagnostic significance. Moreover, substance identification solely based on retention time can be difficult. Implementation of mass spectrometric methods for analysis of amino acids in clinical routine will provide a better analytical platform for identification and quantification of amino acids, as well as 


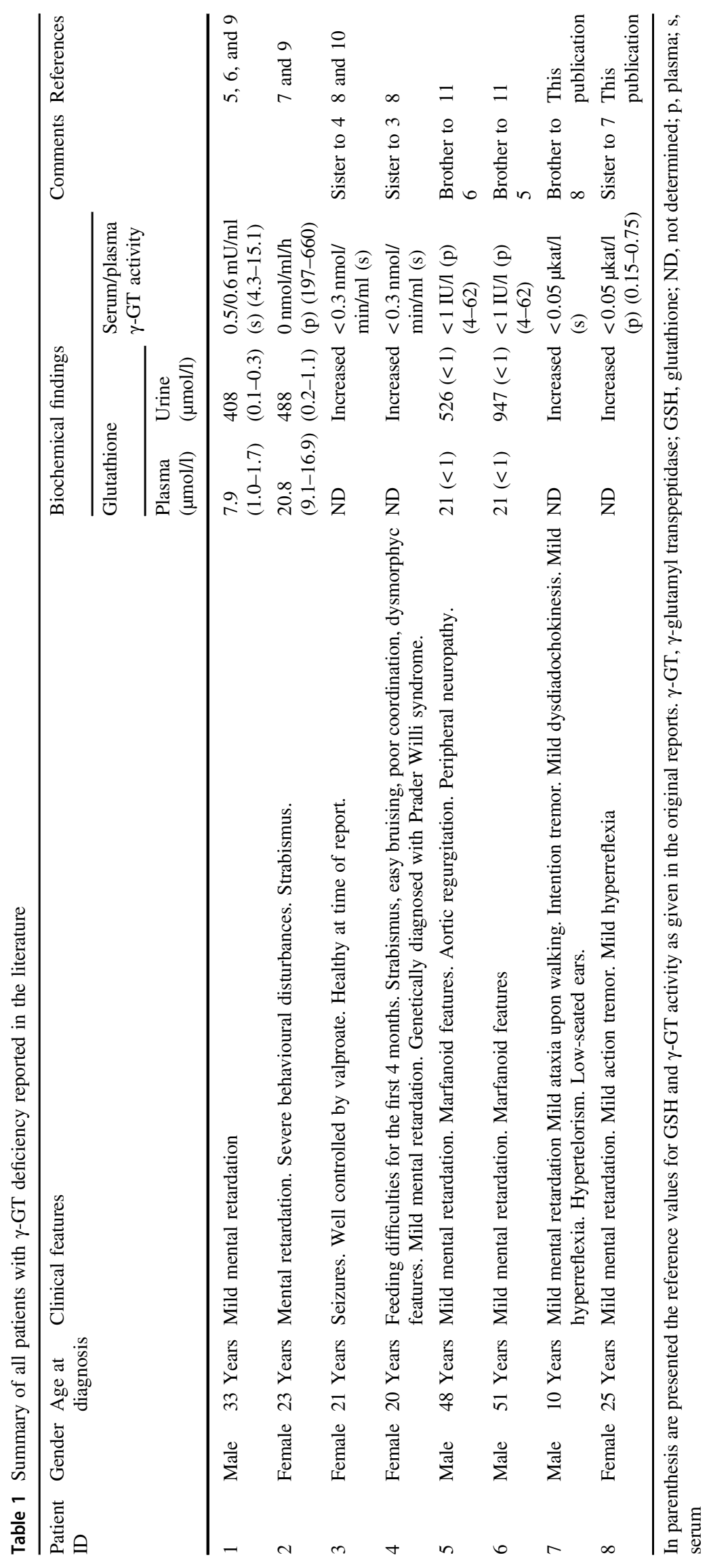


di- and tripeptides with diagnostic value, such as GSH, selecting away other interfering substances [19].

Another key biochemical marker in the diagnosis of this condition is the activity of $\gamma$-GT in serum. However, $\gamma$-GT is mainly used as a marker of liver disease but is not usually included in the screening of metabolic diseases. Glutathionuria has also proved elusive regarding its genetic cause. The presence of a number of genes and pseudogenes sharing high level of homology with GGT1 (see Supplementary Figure 1) and the fact that some regions in GGTI have low complexity have probably contributed to this. Moreover, large deletions are not easy to detect with conventional sequencing methods. It will be very interesting to find out whether large deletions in GGT1 are a common cause for $\gamma$-GT deficiency. It is important to point out that WGS provided all data needed to detect and characterize the reported indel to the nucleotide level, and by doing so it saved considerable time and resources that would have been needed had other "less expensive" sequencing techniques been used in the genetic investigation.

Human GGT1 is mostly expressed in kidney and small intestine. Compared with these, the level of expression in brain is low and yet the main affected organ in all glutathionuria patients reported is the central nervous system. Attempts to address this apparent discrepancy with mice models of $\gamma$-GT deficiency have not entirely succeeded [2023]. These mice present a severe phenotype with dwarfism, cataracts, infertility, and early death as main symptoms. In one report, agitation and tremor after stimulation was observed, although anatomic and histologic examination of the brains did not show any abnormality [21]. Another report suggested that the paucity of symptoms related to the central nervous system in these mice might be explained by their shortened lifespan [23]. Two hypotheses as to the susceptibility of the nervous tissue to alterations in GSH homeostasis have been proposed, namely its role as antioxidant and the possibility of GSH acting as neurotransmitter, either as such or as a source of glutamate, glycine, and cysteine, all three neuroactive substances ([3] and references therein). MRI of the brain of patient 1 in our report has shown clear abnormalities, whereas MRI of patient 2 was normal. As no information of this kind is available from any of the other patients reported in the literature, it will be of great relevance to collect MRI data from other patients to determine whether the alterations found in patient 1 are a hallmark of the disease.

Nucleotide resolution at the deletion junction allows us to speculate about the mechanism causing the deletion reported. Both breakpoints are located on Alu elements: AluSc at the centromeric end and AluSx at the telomeric end (Fig. 4b). Alu-Alu-mediated recombination is a wellknown cause for genomic rearrangements. It was initially attributed to unequal non-allelic homologous recombination
(NAHR), but the general consensus nowadays is that the degree of identity shared between Alu elements is not high enough to support NAHR. Alternatively, microhomologymediated DNA replication-based mechanisms have been proposed ([24] and references therein). Moreover, alignment of both Alu sequences close to the breakpoints (Fig. 4b, central panel) reveals the presence of an area which is highly homologous to the Alu-DEIN consensus sequence, a recombinogenic hotspot [25]. Analysis of the junction also revealed the presence of a $13 \mathrm{bp}$ insertion, identical to two segments of DNA, one on each Alu element, which happens to be part of the Alu-DEIN sequence (Fig. 4b, lower panel). Insertion of short templates originating from areas flanking copy number variation (CNV) junctions has been reported to be as frequent as $35 \%$ of simple CNV junctions and proposed to represent shortdistance template switches due to misalignment or replication slippage within the same replication fork [26]. Based on all this, we propose that the deletion presented here is caused by a two step mechanism involving replication slippage, causing the $13 \mathrm{bp}$ insertion, and microhomologymediated, replication-based template switching between Alu elements, causing the $14.9 \mathrm{~kb}$ deletion. We find it impossible to define the order in which the two events took place, nor which of the two possible regions was the origin of the insertion. Different possibilities, all based on microhomologies ranging from 1 to $4 \mathrm{bp}$, are presented in Supplementary Figure 2. Although all of them are in principle possible, we would like to suggest that the Alu-DEIN sequence present in both Alu elements has a crucial role in bringing both Alu elements in close proximity, thus favoring recombination in that particular region. That would be in consonance with the growing concept that genomic architecture has an important role in guiding recombination events [27]. It is even tempting to speculate that a transient structure between both replication forks interacting at the Alu-DEIN sequence facilitates backward replication slippage at precisely that region (Supplemetary Fig. 2, bottom panel).

In conclusion, we present two siblings with glutathionuria caused by a large deletion in GGT1. This is, to our knowledge, the first report linking a genetic variant to glutathionuria. The heterozygous nature of the healthy parents and the healthy brother reaffirms the autosomal recessive inheritance pattern of this condition. The difficulty to identify GSH in urine with conventional amino acid analyzers, the presence of multiple genes with partial homology with GGT1, and a diffuse clinical picture have possibly hindered the diagnosis of this disease in the past. Extra care in the interpretation of amino acid profiles, hydrolysis of samples presenting extra peaks, or better still, mass spectrometric analysis of amino acids in clinical routine, inclusion of serum $\gamma$-GT activity in the screening of 
metabolic diseases, and the use of WGS as first line of genetic investigation will significantly increase the chances of identifying patients with glutathionuria.

Acknowledgements We thank Donald Runsteen for excellent technical assistance in the analysis of urine amino acids.

Funding This work was partially financed with Laboratoriemedicin Research and Development funds, Sahlgrenska University Hospital, Gothenburg, Sweden.

\section{Compliance with Ethical Standards}

Conflict of interest The authors declare that they have no conflict of interest.

\section{References}

1. Castellano I, Merlino A. Gamma-Glutamyl Transpeptidases: Structure and Function, SpringerBriefs in Biochemistry and Molecular Biology (Springer, 2013).

2. Dickinson DA, Forman HJ. Cellular glutathione and thiols metabolism. Biochem Pharmacol. 2002;64:1019-26.

3. Ballatori N, Krance SM, Notenboom S, Shi S, Tieu K, Hammond CL. Glutathione dysregulation and the ethyology and progression of human diseases. Biol Chem. 2009;390:191-214.

4. O'Daly S. An abnormal sulphydryl compound in urine. Ir J Med Sci. 1968;7:578-9.

5. Goodman SI, Mace JW, Pollack S. Serum gamma-glutamyl transpeptidase deficiency. Lancet. 1971;1:234-5.

6. Schulman JD, Goodman SI, Mace JW, Patrick AD, Tietze F, Butler EJ. Glutathionuria: Inborn error of metabolism due to tissue deficiency of gamma-glytamyl transpeptidase. Biochem Biophy Res Commun. 1975;65:68-74.

7. Wright EC, Stern J, Ersser R, Patrick AD. Gluathionuria: $\gamma$ glutamyl transpeptidase deficiency. $J$ Inherit Metab Dis. 1979;2:3-7.

8. Hammond JW, Potter M, Wilcken B, Truscott R. Siblings with $\gamma$ glutamyl transferase deficiency. $\mathrm{J}$ Inherit Metab Dis. 1995; 18:82-83.

9. Griffith OW, Meister A. Excretion of cysteine and $\gamma$ glutamylcysteine moieties in human and experimental animal $\gamma$ glutamyl transpeptidase deficiency. Proc Natl Acad Sci USA. 1980;77:3384-7.

10. Hammond JW, Potter M, Sim KG, Wilcken B. Reduced glutathione, $\gamma$-glutamylcysteine and $\gamma$-glutamylglutamine in $\gamma$ glutamyltransferase deficiency. $J$ Inherit Metab Dis. 1999;22:235-9.

11. Iida M, Yashuhara T, Mochizuki H, Takakura H, Yanagisawa T, Kubo H. Two Japanese brothers with hereditary $\gamma$-glutamyl transpeptidase deficiency. J Inherit Metab Dis. 2005;28:49-55.
12. Heisterkamp N, Groffen J, Warburton D, Sneddon TP. The human gamma-glutamyltransferase gene family. Hum Genet. 2008;123:321-32.

13. Wickham S, West MB, Cook PF, Hanigan MH. Gamma-glutamyl compounds: substrate specificity of gamma-glutamyl transpeptidase enzymes. Anal Biochem. 2011;414:208-14.

14. West MB, Wickham S, Parks EE, Sherry DM, Hanigan MH. Human GGT2 does not autocleave into a functional enzyme: a cautionary tale for interpretation of microarray data on redox signaling. Antioxid Redox Signal. 2013;19:1877-88.

15. Bui TT, Nitta RT, Kahn SA, et al. $\gamma$-glutamyl transferase 7 is a novel regulator of glioblastoma growth. BMC Cancer. 2015;15:225.

16. Spackman DH, Stein WH, Moore S. Automatic recording apparatus for use in chromatography of amino acids. Anal Chem. 1958;30:1190-206.

17. Ozols J. Amino acid analysis. Methods Enzymol. 1990;182:587-601.

18. Thorvaldsdóttir H, Robinson JT, Mesirov JP. Integrative Genomics Viewer (IGV): high-performance genomics data visualization and exploration. Brief Bioinform. 2013;14:178-92.

19. Piraud M, Vianey-Saban C, Petritis K, et al. ESI-MS/MS analysis of underivatised amino acids: a new tool for the diagnosis of inherited disorders of amino acid metabolism. Fragmentation study of 79 molecules of biological interest in positive and negative ionization mode. Rapid Commun Mass Spectrom. 2003;17:1297-311.

20. Lieberman MW, Wiseman AL, Shi ZZ, et al. Growth retardation and cysteine deficiency in gamma-glutamyl transpeptidase-deficient mice. Proc Natl Acad Sci USA. 1996;93:7923-6.

21. Harding CO, Williams $\mathrm{P}$, Wagner $\mathrm{E}$, et al. Mice with genetic gamma-glutamyl transpeptidase deficiency exhibit glutathionuria, severe growth failure, reduced life spans, and infertility. J Biol Chem. 1997;272:12560-7.

22. Will Y, Fischer KA, Horton RA, et al. Gammaglutamyltranspeptidase-deficient knockout mice as a model to study the relationship between glutathione status, mitochondrial function, and cellular function. Hepatology. 2000;32:740-9.

23. Yamada K, Tsuji T, Kunieda T. Phenotypic characterization of Ggt1(dwg/dwg) mice, a mouse model for hereditary $\gamma$ glutamyltransferase deficiency. Exp Anim. 2013;62:151-7.

24. Carvalho CM, Lupski JR. Mechanisms underlying structural variant formation in genomic disorders. Nat Rev Genet. 2016;17:224-38.

25. Rüdiger NS, Gregersen N, Kielland-Brandt MC. One short well conserved region of Alu-sequences is involved in human gene rearrangements and has homology with prokaryotic chi. Nucleic Acid Res. 1995;23:256-60.

26. Carvalho CM, Pehlivan D, Ramocki MB, et al. Replicative mechanisms for $\mathrm{CNV}$ formation are error prone. Nat Genet. 2013;45:1319-26.

27. Anand RP, Tsaponina O, Greenwell PW, et al. Chromosome rearrangements via template switching between diverged repeated sequences. Genes Dev. 2014;28:2394-406. 